\title{
SIMULACIÓN DE UNA RED MÓVIL 5G EN LA CIUDAD DE BOGOTÁ
}

\author{
Jonathan Leonardo Buitrago Arias dol ,Darwin Sneyder González Acuña. \\ Universidad Autónoma de Colombia, Bogotá, Colombia
}

Recibido: 08/10/2020 Revisado: 15/10/2020 Aceptado: 15/10/2020 Publicado: 28/01/2021

\section{Resumen}

El presente proyecto explora la red 5G en base a los estándares que propone la UIT para la implementación de la nueva red móvil y se pone en práctica las características más relevantes. Se evidencian sus alcances y limitaciones por medio de simulaciones de cobertura en las cuales se observa el comportamiento de esta red en diferentes escenarios de la ciudad de Bogotá, en las simulaciones se configuran diferentes parámetros entre los cuales se encuentran las bandas de frecuencias, áreas de simulación, coberturas, parámetros de radio transmisor, antenas, métodos de estudio, entre otros. A partir de la variación de los parámetros se obtienen resultados de las simulaciones con diferentes comportamientos los cuales se comparan y se analizan para identificar sus diferencias y similitudes de esta manera se identifica los parámetros que influyen de mejor manera a el estudio de la cobertura, estas simulaciones se realizan con la ayuda del software Xirio Online, el cual es una herramienta enfocada en simulaciones profesionales que aporta resultados con una alta precisión. Además, de analizar los resultados y mostrar el comportamiento de la red este proyecto quiere contribuir al estudio de la red móvil 5G e impulsar su implementación en el país.

\section{Palabras Clave}

Simulación de redes, red 5G, coberturas de propagación, parámetros de simulación, bandas de frecuencias.

\begin{abstract}
This project explores the $5 \mathrm{G}$ network based on the standards proposed by the ITU for the implementation of the new mobile network and the most relevant characteristics are put into practice. Its scope and limitations are evidenced by means of coverage simulations in which the behavior of this network is observed in different scenarios of the city of Bogotá, in the simulations different parameters are configured among which are the frequency bands, areas of simulation, coverage, radio transmitter parameters, antennas, study methods, among others. From the variation of the parameters, results of the simulations with different behaviors are obtained, which are compared and analyzed to identify their differences and similarities.In this way, the parameters that best influence the study of coverage are identified. Simulations are carried out with the help of the Xirio Online software, which is a tool focused on professional simulations that provides highly accurate results. In addition, by analyzing the results and showing the behavior of the network, this project wants to contribute to the study of the $5 \mathrm{G}$ mobile network and promote its implementation in the country.
\end{abstract}

\section{Keywords}

Network simulation, 5G network, propagation coverage, simulation parameters, frequency bands. 


\section{Introducción}

La presente investigación lleva como título Simulaciones de cobertura de un canal 5G en escenarios de radio propagación de la ciudad de Bogotá D.C, este estudio hace parte del semillero de investigación SITEL a cargo del Ingeniero Javier Enrique Arévalo Peña de la Fundación Universidad Autónoma de Colombia, el cual pretende investigar acerca de la red 5G por medio de la simulación de cobertura en las diferentes zonas de la capital utilizando diferentes software de simulación.

La red 5G es la próxima generación de redes móviles, ofrece nuevas aplicaciones y servicios ya que posee mejores características que tiene la actual red 4G como lo son las bajas latencias que hace que los tiempos de respuesta sean más cortos generando mejores comunicaciones, esta red debido a sus altas velocidades permite abrir las puertas a mejorar la experiencia de los usuarios encaminándolos hacia una nueva era de la tecnología. La red 5G brinda un apoyo fundamental a los ciudadanos, empresas y a los gobiernos que, contribuyendo a la formulación de política responsable, para transformar sus ciudades en ciudades inteligentes y facilite que las personas y los miembros de estas ciudades descubran y aprovechen los beneficios que la red trae consigo.

Conocer la red 5G es importante por eso nace la necesidad de realizar investigaciones del uso de esta red en la ciudad, ya que Bogotá es la capital del país y es una ciudad la cual ofrece oportunidades para sus habitantes y donde migra la mayor parte de personas en busca de un futuro mejor. Por medio de las simulaciones que se van a realizar se prende conocer los alcances, ventajas y desventajas que puede ocasionar una futura implementación de esta red en la ciudad. Mediante el software de simulación se pretende ilustrar los sistemas de comunicación de la red 5G.

Las simulaciones se realizarán a partir de las sugerencias técnicas establecidas por la MINTIC en su informe sentando las bases para las redes 5G oportunidades y desafíos ITU. (2018). En donde se brinda un enfoque práctico y cometido a los responsables de la formulación de políticas y reglamentaciones técnicas para implementación de la 5G, entre las cuales se utilizará la frecuencia

$3.5 \mathrm{GHz}$ con la que se analizará el espectro radio eléctrico y el nivel de cobertura.

\section{Planteamiento del problema}

La tecnología es una herramienta importante para el ser humano a medida que este avanza se crean nuevas necesidades, por eso la importancia de mejorar las redes comunicaciones, con el fin de satisfacer estas nuevas demandas, para poder implementarlas se deben realizar simulaciones de la nueva red con el fin de conocer acerca de su funcionamiento, aportes, alcances y limitaciones, para a partir de esto resultados tener una idea más clara del porque es necesario mejorar la red.

Los Software de simulaciones son una herramienta que permite analizar procesos, realizar mejoras y anticipar soluciones, por eso en el proyecto de investigación se utilizara este recurso donde se sitúan los parámetros sugeridos por la división y desarrollo de las tecnologías y redes de telecomunicaciones (UIT) como base para la implementación de una canal 5G en la ciudad de Bogotá.

Para tratar de lograr tratar de lograr las metas que se plantea la red $5 \mathrm{G}$ se van a incluir nuevas bandas de frecuencias con el objetivo de conseguir incrementar el ancho de banda, concretamente se van a usar bandas de ondas milimétricas por encima de $3.5 \mathrm{GHz}$, las cuales mejoran el alcance de la señal al cual se propaga, se disminuyen los retardos de respuestas debido a su baja latencia, y contribuye a una reducción del $90 \%$ del 
consumo de la energía de la red, todas estas características contribuyen a que con la red $5 \mathrm{~g}$ hallan más dispositivos conectados por unidad de área.

La dirección de esta investigación es simular la cobertura de una canal 5G nace de varios factores los cuales afectan las redes móviles actuales puesto que la necesidad de requerir más recursos para el tránsito de datos es cada vez mayor, la red $4 \mathrm{G}$ suministra velocidad pero no alcanza a cubrir en su totalidad, por ende se piensa en crear una nueva red la cual supla las necesidades actuales, se puede evidenciar los beneficios y alcances de esta por medio de las simuladores los cuales nos permitan visualizar los diferentes factores que la red 5G puede apoyar, teórica mente se establecen unas características las cuales superen a la red actual, como lo son coberturas, conexiones masivas, velocidades entre otras.

En la práctica se tienen que realizar simulaciones para evidenciar los diferentes factores que la red 5G pretende mejorar, el uso de los simuladores nos brinda un apoyo fundamental para tener idea global de implementación de dicha red en las ciudades, por esto se verificaran diferentes datos dentro de los simuladores para encontrar la mejor forma de implementar la nueva red.

Las tecnologías desarrolladas cada día requieren de comunicaciones más efectivas, así como lo menciona la web REMCOM donde se evidencia un uso de comunicaciones inalámbricas e innovaciones para lograr así obtener niveles de radio frecuencias que viajen a altas velocidades para así proporcionar datos con latencias más rápidas (REMCOM, 2020).

A su vez la nueva red 5G contribuye a los sectores de la salud realizando un monitoreo de datos el cual puede ser enviado desde la hoja por las facilidades de la utilización de la información, a la educación en cuanto a la tecnología y el bit data facilitando la descarga de contenido de una forma más rápida y la utilización de nueva tecnología educativa permitiendo que los estudiantes tengan acceso más fácil a la información.

El sector energético será uno de los más impulsados ya que se fomenta la utilización de las energías alternativas y los alumbrados inteligentes, en cuanto a la industria haciendo que se pueda automatizar de forma completa toda una planta, en cuanto al área rural por medio de la utilización de tecnología que ayuda a cuidar los cultivos y en cuanto a la movilidad un mejor manejo ya que va a hacer un seguimiento permanente y se relacionara con una base de datos.

\section{Objetivos}

\section{Objetivo General:}

- Llevar a cabo un estudio de simulaciones de cobertura de un canal 5G en escenarios de radio propagación de la ciudad de Bogotá D.C Objetivos Específicos:

- Realizar una revisión bibliográfica para determinar las características principales de los sistemas móviles $5 \mathrm{G}$.

- Efectuar simulaciones de cobertura con una herramienta de software especializada de un canal 5G en diferentes áreas de Bogotá.

- Analizar los resultados de las simulaciones de cobertura para evidenciar el desempeño de radio propagación del canal 5G en los escenarios de radio propagación estudiada. Metodología 


\section{Planificación}

La tecnología avanza rápidamente con el fin de dar solución a muchos de nuestros problemas y no solo eso también nos ayuda a realizar nuestras actividades de una mejor manera, por esto se requiere de una generación la cual pueda mejorar el sostenimiento para nuestra cobertura. Para esto hay que mejorar diferentes aspectos ya conocidos en la generación 4G, Se tendrá que estudiar y analizar la siguiente generación la cual mejorara las coberturas y las velocidades de respuesta de envíos de datos, una generación que se acople a una conexión masiva sin tener pérdidas de latencia y mejorando la banda ancha móvil.

\section{Ejecución}

Se realizan varias simulaciones de cobertura de un canal 5G en la ciudad de Bogotá, para observar y analizar qué tan viables son sus respuestas, una simulación que abrirá camino a pruebas de mejoras en cuanto a propagación y respuestas en tiempo real. Comparación de datos teóricos con datos prácticos para tener un margen de error mínimo y dar por certeza que la generación 5G es la gran solución para los nuevos desafíos de la comunicación y los puntos estudiados en este seminario.

\section{Finalización}

Realizar el estudio de las simulaciones desarrolladas en la ciudad de Bogotá y de a partir de estos resultados, poder dar una idea más concreta de los beneficios que puede traer la implementación de esta red en la ciudad de Bogotá. De la misma manera conocer las desventajas que pueda presentar esta red, con este análisis de resultados poder afirmar si necesaria o no la implementación de un canal de cobertura $5 \mathrm{~g}$ en la ciudad.

\section{Hallazgos}

La red 5G es la nueva red móvil que pretende mejorar todos los aspectos de la actual red 4G y 4.5G con características que cada día se requieren corregir, estos nuevos beneficios que nos aporta esta nueva red logran aumentar la capacidad de conectividad lo que en las redes actuales muy común mente se suelen colgar por la densidad de conexiones masivas que pueden llegar a ocurrir, las transmisiones de datos se verán aumentadas debido a las altas latencias que puede alcanzar la red 5G y la capacidad de velocidades aumentadas entorno los $10 \mathrm{Gbps}$ lo que sería hasta 100 veces mayor a las redes actuales, además de la disponibilidad de trabajar acorde con las nuevas tecnologías como lo son el internet de las cosas y la nube generando beneficios en todos los aspectos.

Para lograr disponer de la red móvil 5G se tendrán que realizar simulaciones con las que se verán la disponibilidad en entorno a la ciudad de Bogotá, dando así una demostración de las de estudios de coberturas que permitirán evidenciar los alcances que puede lograr en ciudades las cuales se disponen a implementar redes con mejores beneficios.

El Software Xirio Online brinda la oportunidad de realizar simulaciones con características específicas las cuales favorecen el medio en el cual se requiere realizar dicho estudio además para poder entender la nueva red que se planea incorporar en la ciudad de Bogotá, para esta ocasión se pretende entender diferentes aspectos que puede aportar la nueva red 5G en la ciudad como lo son las coberturas, la frecuencia, parámetros de las antenas y sobre todo el funcionamiento del software para poder emplear un cálculo de simulación adecuado. 
Inicialmente Xirio Online es un software de pago con el cual se podrá realizar estudios profesionales tanto en sitios urbanos como rurales, con la disponibilidad de cartografías precisas para poder así tener simulaciones más adecuadas a lo que se necesite, tiene dos formas para poder iniciar los estudios una con pago que brinda la opción de entrar en más detalle los estudios finales para observar coberturas pero de igual modo brinda la posibilidad de un modo de cálculo gratuito el cual también es válido para la planificación de estudios iniciales, da soporte con un manual completamente extenso con el que da apoyo para realizar los estudios adecuada mente.

$\mathrm{Al}$ iniciar un estudio brinda unas opciones del tipo de estudio, en la figura 1 se observa los diferentes estudios que se pueden utilizar entre los cuales se encuentran: estudios de enlace, cobertura, cobertura de interior, cobertura multitransmisión y red de transporte, cuando ya se tiene defino el tipo de estudio que se va realizar en el caso de estudio de cobertura 5G se pasa a seleccionar un servicio o tecnología, para el estudio cobertura que se pretende estudiar es la servicio móvil con subcategoría de $5 \mathrm{G}$ y servicio $5 \mathrm{G}$ a partir de este punto el simulador brinda la posibilidad de realizar un estudio en modo asiste, el cual es el recomendado para así agregar ciertos parámetros los cuales permitirán incorporar datos adecuados para que el estudio no genere errores o por el contrario da la opción de aceptar y se comienzan a agregar los parámetros que se deseen.

El software tiene soporte con cartografías con alta definición para así realizar las simulaciones en entornos más reales y más precisos para el servicio móvil de red 5G la posición será en la ciudad Bogotá, una ciudad la cual pretende avanzar en su red móvil dando beneficios a las nuevas tecnologías que cada día requieren más características para lograr un funcionamiento más eficiente.

La sectorización es importante ya que permite definir sectores en la misma estación, y crear estudio de cobertura para cada uno de los sectores entre más sectores se agreguen en el estudio se evidenciará una cobertura más completa. En la figura 2 se observa el proceso realizado para la sectorización del estudio donde en el ejemplo se realiza un estudio con 3 sectores en los cuales se definen las áreas de trabajo de la antena.

Los parámetros del tipo de sector se realizan de forma estándar para los servicios radioeléctricos, con una altura de la antena de 30 metros lo que en condiciones normales se realizan con antenas en el país.

El software Xirio Online cuenta con una gran lista de bandas de frecuencias para el estudio de la red 5G las cuales se pueden visualizar en la figura 3, el estudio se enfocara en una frecuencia baja sobre los 3.5Ghz por lo cual es importante ajustar el parámetro sobre esta banda con una frecuencia inicial entre los $3.5 \mathrm{GHz}$ y una frecuencia final de $3.6 \mathrm{GHz}$ en el tramo inferior y banda frecuencia inicial entre los $3.7 \mathrm{GHz}$ y una frecuencia final $3.8 \mathrm{GHz}$ en el tramo superior como se observa en la figura 4, este parámetro con el fin de mantener un margen a la frecuencia que se pretende trabajar, la potencia es una característica necesaria para determinar la propagación que puede generar la antena por ende se realiza el estudio con una potencia de $5 \mathrm{~W}$ y otros parámetros que se pueden variar dependiendo al estudio a realizar, como los parámetros del radio como lo muestra la figura 5 .

El método de estudio que Xirio Online brinda la posibilidad de realizar simulaciones basadas en modelos estándares de carácter internacional, figura6, los cuales tienen la posibilidad de configurar determinados 
parámetros para ajustar las condiciones a las que se van a trabajar dependiendo de las propagaciones a las que se pretende simular una cobertura, estos métodos que Xirio Online brinda traen consigo parámetros ya predefinidos lo cual facilita más su implementación puesto que solo se tiene que tener en cuenta la frecuencia a trabajar, para la red móvil 5G los métodos de cálculos que se pueden utilizar son varios como se observa la figura 5 y la figura 6, pero para esta ocasión se utilizara el de New York University debido a que su rango de frecuencias está dentro de la red 5G.

El resultado del estudio de la simulación de cobertura se podrá observar por medio de múltiples colores como en la imagen figura 7 los rangos establecidos para cada color de propagación de la señal dela antena para así tener una perspectiva más clara de lo que nos brinda la red 5G en la ciudad de Bogotá, antes de realizar el cálculo del estudio se tiene que tener presente la incorporación de los sistemas MIMO esto lo que permite es obtener tasas altas de datos mediante la utilización de múltiples canales en paralelo, la ganancia de MIMO DL brinda técnicas en sentido descendente y MIMO UL técnicas en sentido ascendente. En esta simulación se empleó un sistema MIMO DL 2x2 con una ganancia de 1.75 y un MIMO UL 1x2 con ganancia de 1.75 así como se muestra en la figura 8 , además de poder implementar otros parámetros adicionales para mejorar la cobertura de la simulación.

Una vez definidos los parámetros se crean las carpetas de estudio, en la figura 9 hay una capeta central y tres subcarpetas las cuales llevan el nombre de los sectores de cada sector utilizado, si por algún motivo durante la configuración de este estudio se coloca un dato o se desea variar algún parámetro proporcionado se puede cambiar dando click en la configuración de cada sector.

Ya configurados todos los parámetros se calcula el estudio el cual es mostrado en una cartografía donde se ilustran las antenas y sectores utilizados en el escenario escogido en la figura 10 y figura 11 se puede observar el resultado de la distancia de la propagación la cual se muestra por medio de los colores anterior mente mencionados a partir de estas imágenes se pueden sacar las conclusiones necesarias del comportamiento de la red y analizar los resultados.

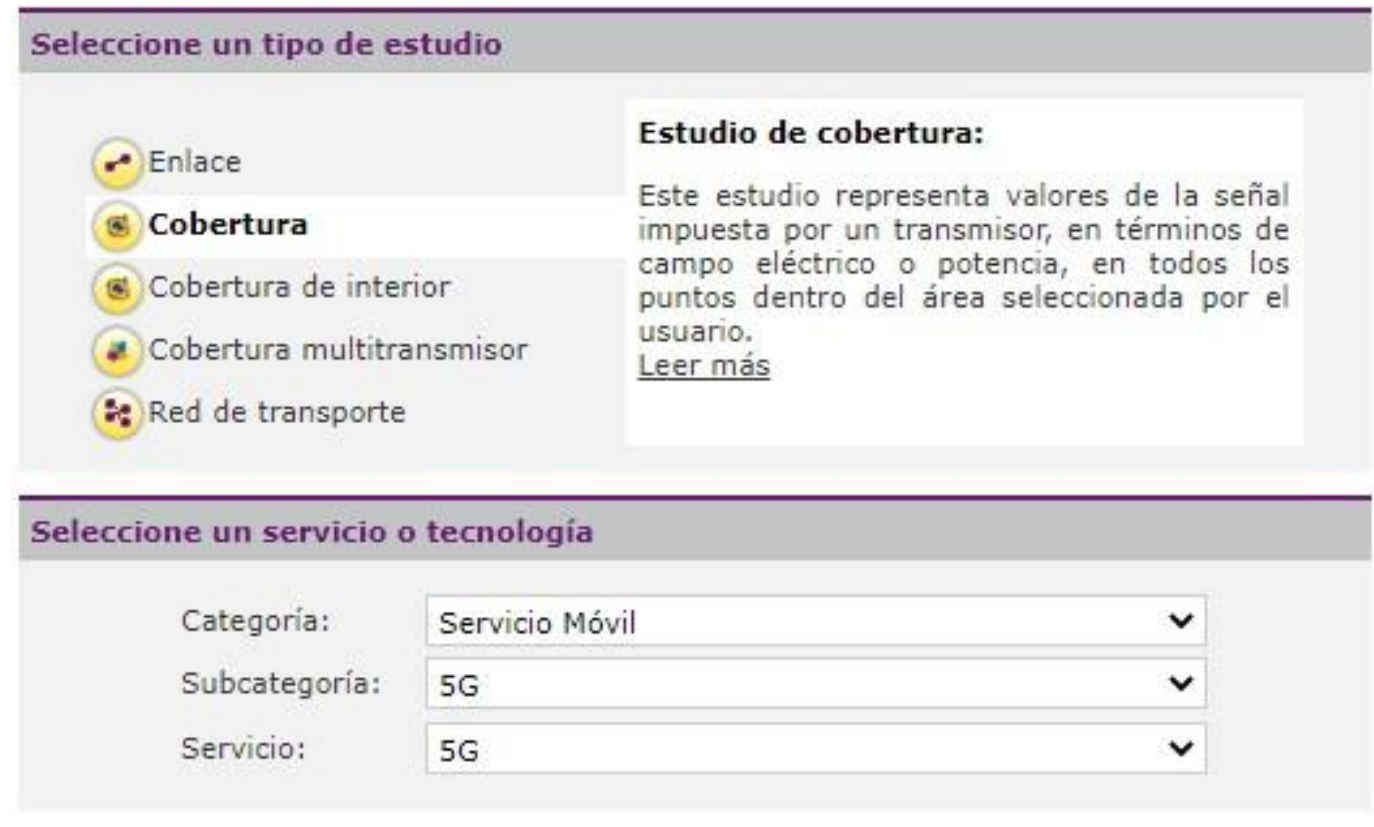

Figura 1. Tipos de estudios 


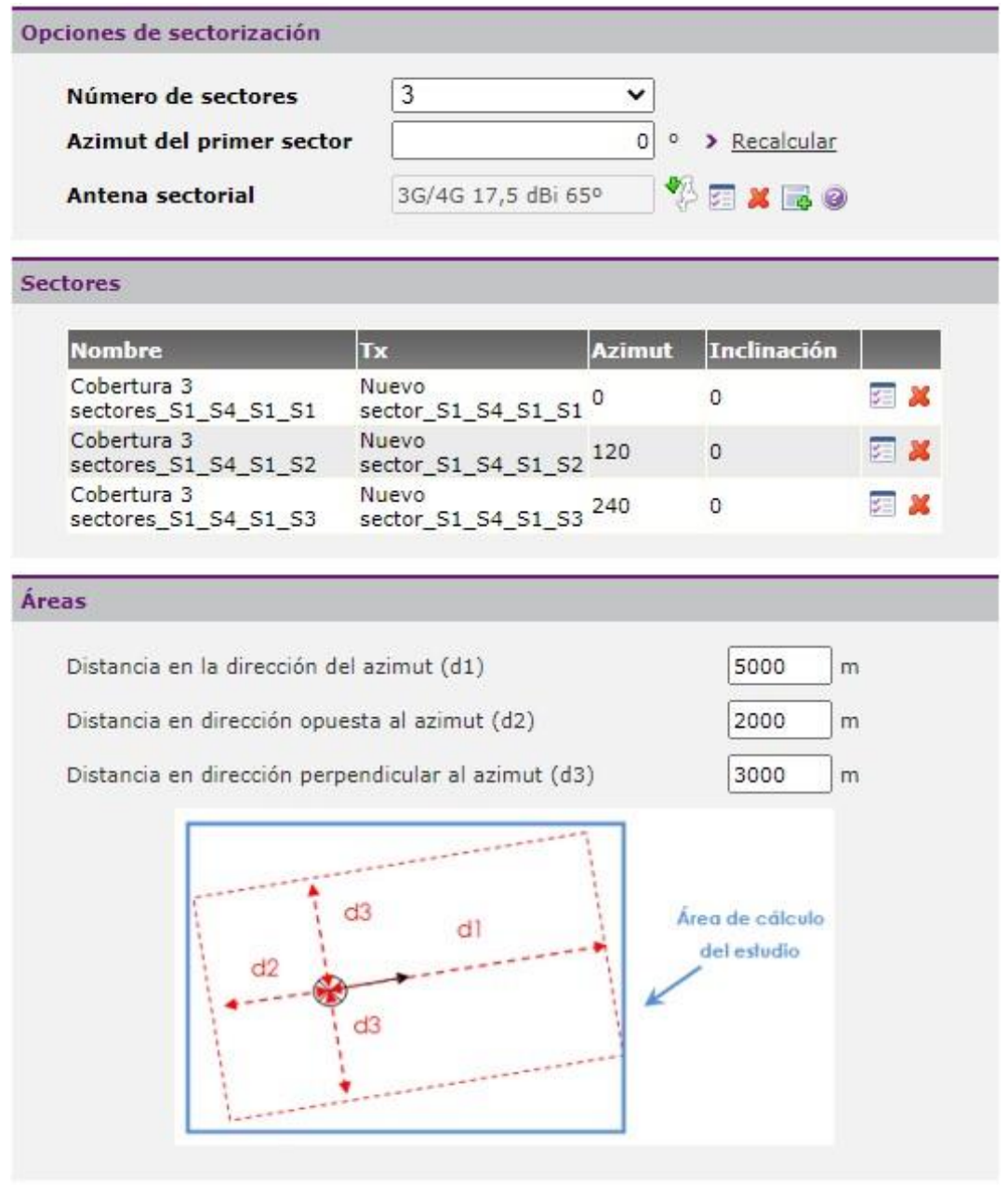

Figura2. Áreas de cálculo.

\begin{tabular}{|c|c|c|c|c|}
\hline 居 圆 & $\widehat{\omega}$ & Band $-27,5 \mathrm{GHz}-7 \mathrm{MHz}$ & Punto-Punto $-27,5 \mathrm{GHz}-7 \mathrm{MHz}$ & Banda $27,5-29,5$ G Servicio Fijo \\
\hline 圆圂 & 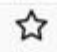 & Band $-3,6 \mathrm{GHz}$ & Punto-Punto $-3,6 \mathrm{GHz}$ & Banda 3600-4200 I Servicio Fijo \\
\hline 圂家 & $\hat{\imath}$ & Band $-3,6 \mathrm{GHz}$ & Punto-Punto $-3,6 \mathrm{GHz}$ & Banda 3600-4200 I Servicio Fijo \\
\hline
\end{tabular}

Figura 3. Bandas de Frecuencia 


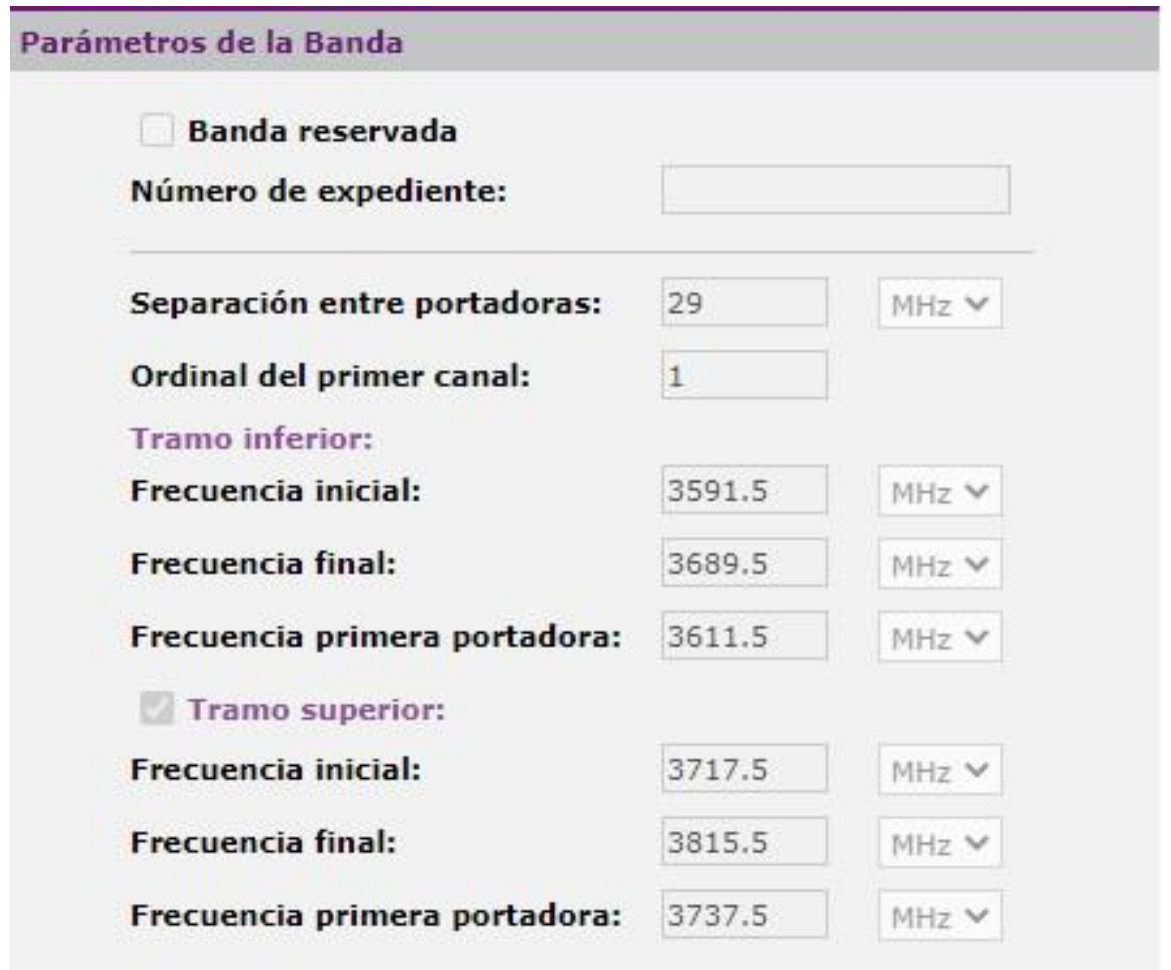

Figura 4. Parámetros de bandas de frecuencias. 


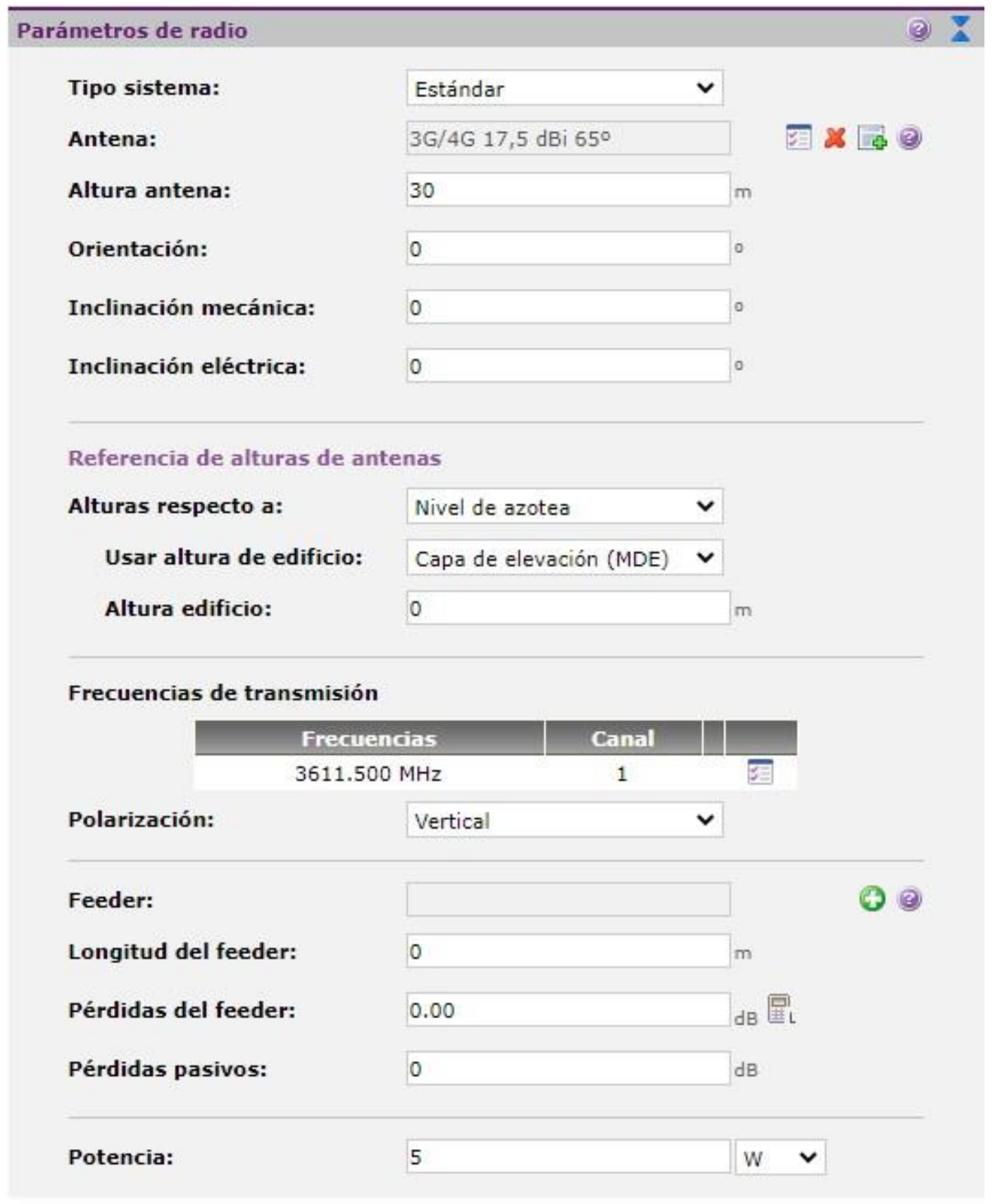

Figura 5. Parámetros de radio. 


\section{Método de cálculo}

Nombre:

Método de propagación:

Resolución:
Nuevo New York University

\section{New York University}

\begin{tabular}{l|l}
100 & $\mathrm{~m} / \mathrm{pixel}$
\end{tabular}

Limitar la distancia máxima del cálculo

Límite:

0

m

Figura 5. Métodos de cálculos.

\begin{tabular}{l} 
New York University \\
\hline Rec. UIT-R P.526-11 \\
\hline Deygout \\
Línea de vista \\
Okumura-Hata \\
Xia-Bertoni \\
Editable \\
Rec. UIT-R P.1546 \\
COST 231 Walfish-Ikegami \\
Rec. UIT-R P.1411-9 \\
Okumura-Hata Modificado \\
Stanford University Interim \\
Rec. UIT-R P.526-13 \\
5GCM \\
3GPP TR 38.901 \\
METIS estocástico \\
mmMAGIC \\
New York University \\
Longley-Rice \\
\hline H-i..---iL.
\end{tabular}

Figura 6. Variables de estudio.

\begin{tabular}{|c|c|c|c|}
\hline Color & Rango & Descripción & \\
\hline & {$[-112.00,-102.00) \mathrm{dBm}$} & & 国 8 \\
\hline & {$[-102.00,-92.00) \mathrm{dBm}$} & & Ex $x$ \\
\hline & {$[-92.00$, Infinity $) \mathrm{dBm}$} & & E 8 \\
\hline
\end{tabular}

Figura 7. Color de los rangos de cobertura. 


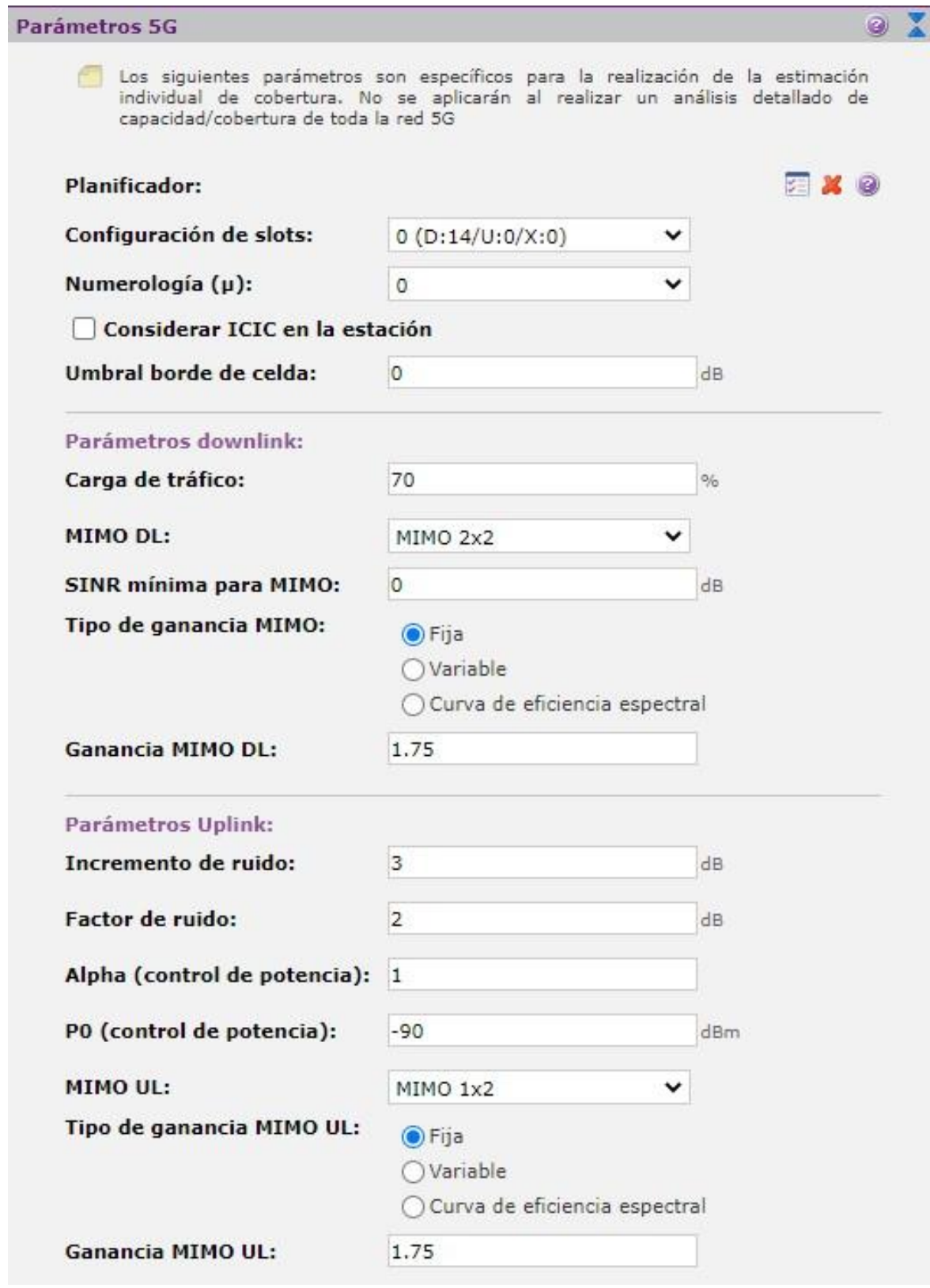

Figura 8. Parámetros 5G. 


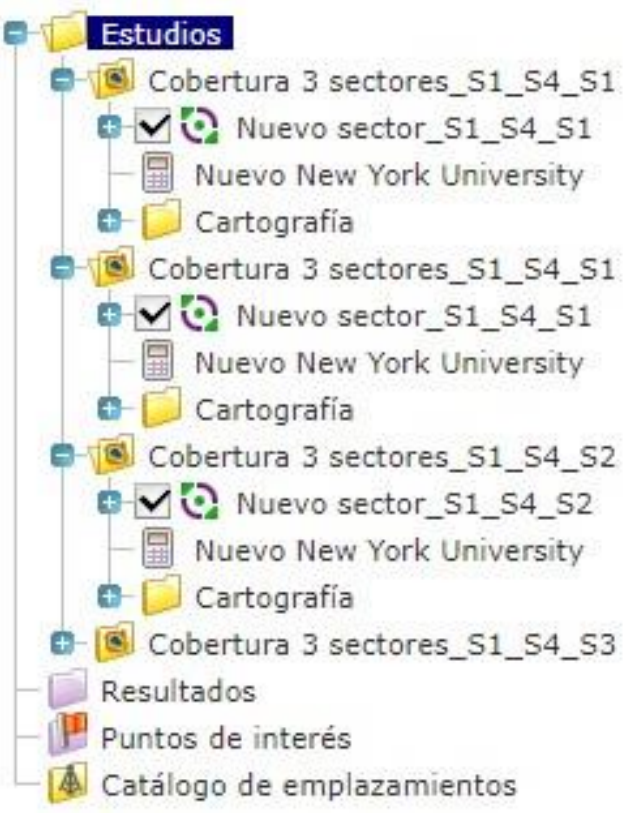

Figura 9. Carpetas de estudios

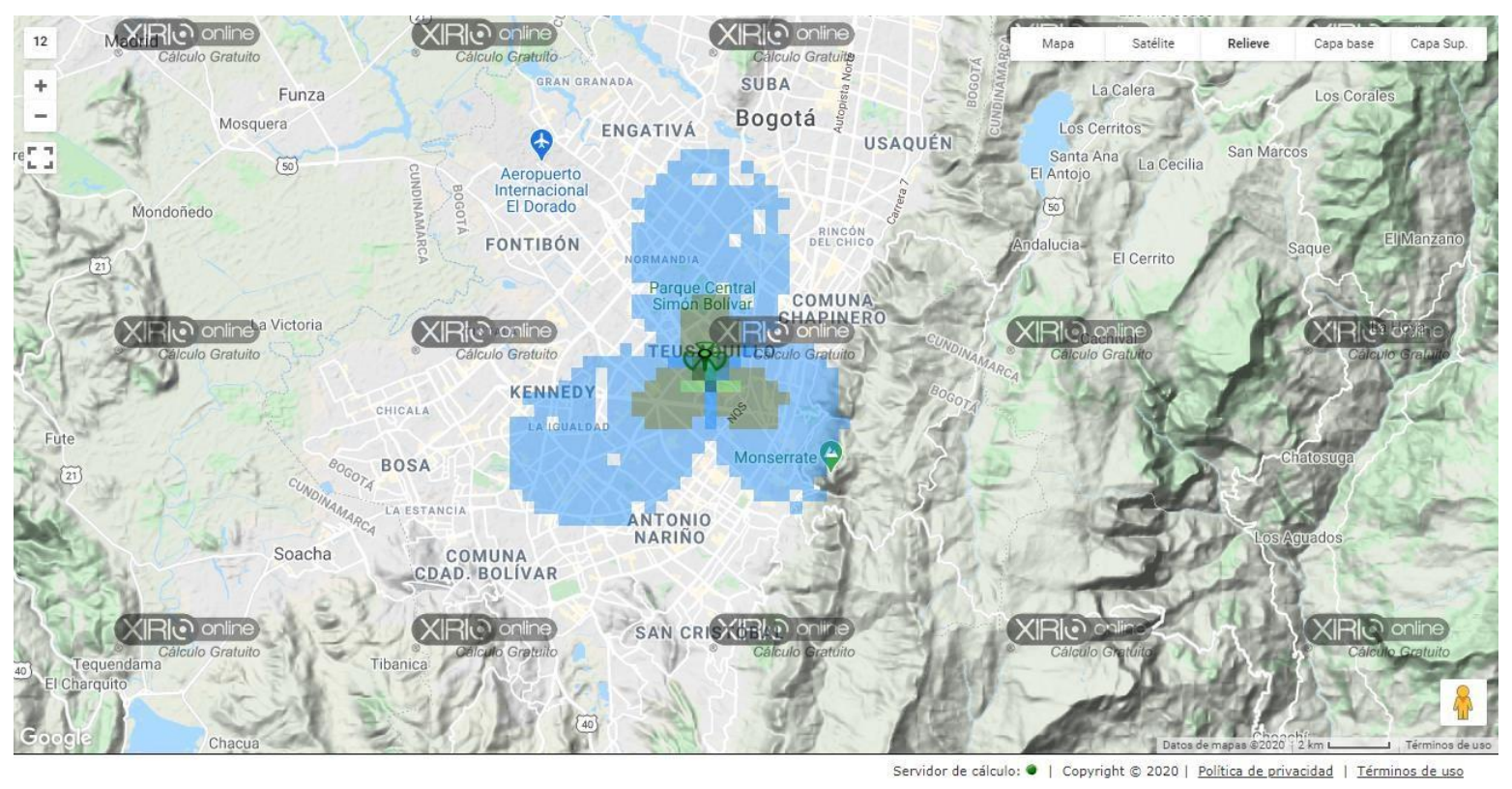

Figura 10. Cobertura red 5G ciudad de Bogotá. 


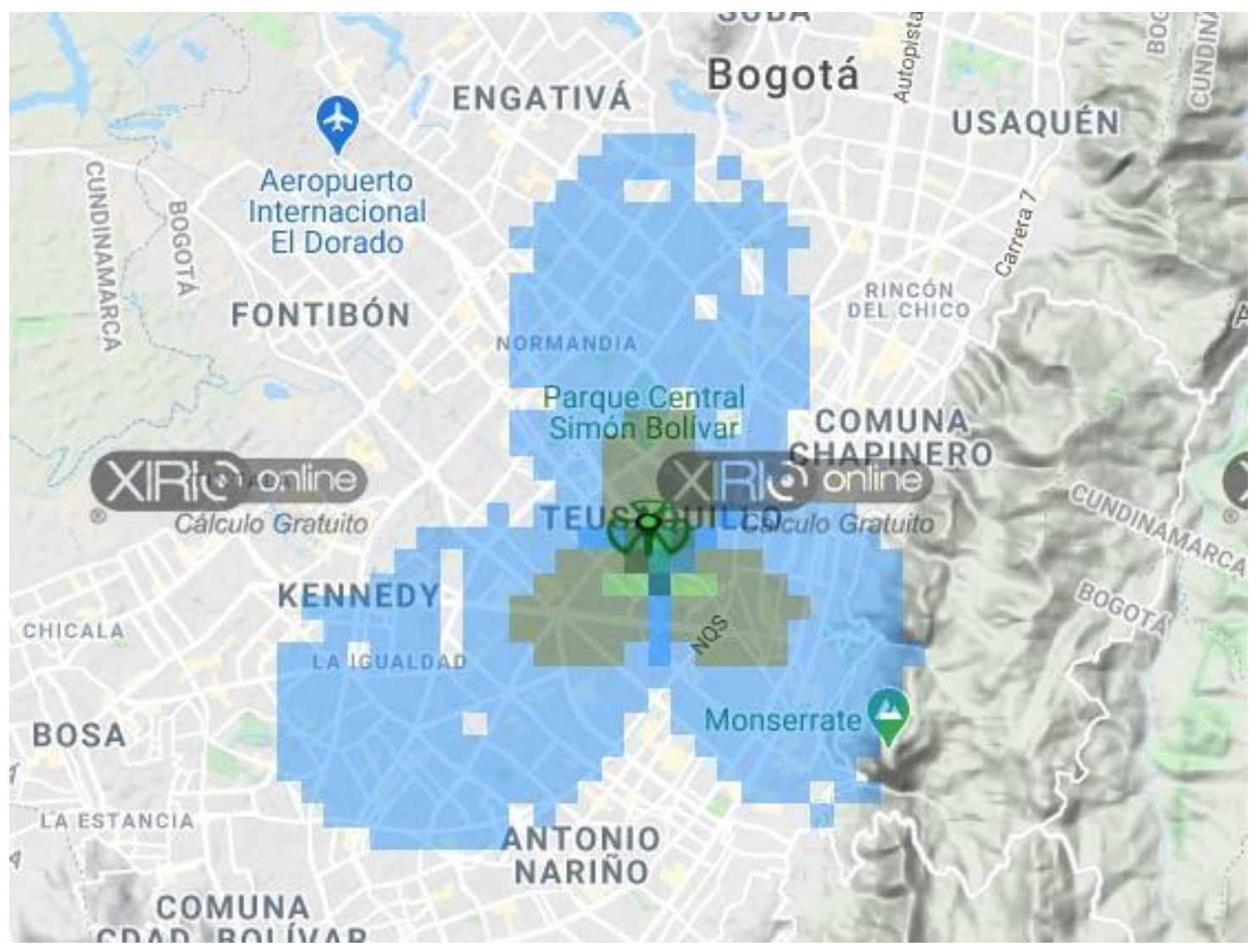

Figura 11. Cobertura Red 5G ciudad de Bogotá.

\section{Contribuciones}

Dentro del avance y el surgimiento de nuevas tecnologías se ha ido buscando nuevas maneras de solucionar los problemas que se nos presentan, una herramienta fundamental en el avance tecnológico han sido las simulaciones aportan considerablemente al estudio de estas redes de telefonía dado que por medio de estas, se puede observar el comportamiento que podría tener una red después de su implementación, estos softwares de simulaciones ayudan también a reducir costos, ya que con ellos se pueden realizar pruebas y montajes virtuales que son rápidos y sencillos.

Dentro de los principales hallazgos del estudio de las redes móviles hecho por parte de la CRC indica que "se evidenció que las redes 5G aportarán mejores niveles de cobertura, calidad de servicio e incentivarán el desarrollo económico, todos estos aspectos de gran relevancia para el país" (Forbes Staff,28/05/2020).

Uno de los principales organizamos que ha intervenido en la investigación y en la búsqueda de la implementación de estas rede es el Ministerio de las tecnologías de la información (MINTIC)quien estableció parámetros con los que debe contar esta nueva red. "en su artículo plan $5 \mathrm{~g}$ Colombia establece bandas de frecuencia para la red $5 \mathrm{~g}$ entre la que especifica que: El espectro inferior a $1 \mathrm{GHz}$ será utilizado para extender la cobertura de la banda ancha móvil 5G de alta velocidad a zonas urbanas, suburbanas y rurales para así contribuir al sustento de los servicios de Internet de las Cosas: la expansión de los servicios 5G, más allá de 
los centros urbanos y dentro de los edificios, no será fácil sin este tipo de espectro radioeléctrico. En la actualidad, hay espectro móvil en este rango que podría ser utilizado para algunos casos en el futuro". (MINTIC, 2019).

"El Departamento Nacional de Planeación realizó un análisis del impacto que podrían tener incrementos en la penetración de Internet con una adecuada calidad del servicio (es decir, con velocidades mínimas de descarga de $10 \mathrm{Mbps}$ ) sobre la desigualdad de ingresos en Colombia, medido con el índice GINI. (DNP, 2018) Una de las más importantes conclusiones del estudio del DNP, es que el incremento de 50 puntos porcentuales en la penetración de Internet para los quintiles de ingresos 1 y 2, puede generar reducciones en el índice GINI entre $0,30 \%$ y $1,26 \%$. Estos valores son bajos, pero significativos pues se traduce en una disminución de la desigualdad de ingresos y lo cual contribuye a cerrar las brechas sociales.” (MINTIC, 2019)

\section{Conclusiones}

Por medio de este software se pueden observar resultados de una simulación de red móvil 5G, el software también muestra un gran potencial para realizar distintos trabajos como el simulado para este trabajo, se logra obtener una cobertura adecuada donde demuestra el nivel alto de cobertura que tendría la red 5G en la ciudad de Bogotá, con la implementación de una antena estándar manejando frecuencias inicialmente de 3.5Ghz, una potencia de $5 \mathrm{~W}$ y una potencia de transmisión de $5 \mathrm{dBm}$ para demostrar su alcance, se observa en la toma de imágenes una buena zona de cobertura permitiendo así con una sola antena cubrir gran parte de Bogotá, se sabe que no sola una antena puede llegar a cubrir toda una ciudad pero se ve que tiene un gran potencial para su implementación con bandas de frecuencias bajas, el software es amigable para realizar distintos estudios permitiendo agregar diferentes parámetros los cuales ayudan a mejorar las condiciones del estudio.

En cuanto a los datos obtenidos la red 5G puede llegar a mejorar gran parte de la cobertura de la ciudad de Bogotá puesto que cada vez se requiere una red que soporte las condiciones que las actuales redes ya no pueden por ende este estudio quiere demostrar que la red 5G tiene varios puntos a favor para ser implementada, con los diferentes beneficios como lo son sus bajas latencias y la mejora de las bandas anchas esta red puede satisfacer la llegada de la industria 4.0. Referencias

- El software de simulación 5G y mimo. (2020). EEUU. REMCOM. https://webcache.googleusercontent.com/search?q=cache:O6DKHfRGCw0J:https://es.rm com.com $/ 5 \mathrm{~g}-\mathrm{mimo}+\& \mathrm{~cd}=1 \& \mathrm{hl}=\mathrm{es}-419 \& \mathrm{ct}=\mathrm{clnk} \& \mathrm{gl}=\mathrm{co}$

- ITU. (2018). Ginebra Suiza. Sentando las bases para la 5g: Oportunidades y desafíos. https://www.itu.int/dms_pub/itu-d/opb/pref/D-PREF-BB.5G_01-2018-PDF-S.pdf

- Forbes Staff. (28/05/2020). 'Colombia tiene las condiciones para impulsar el 5G': CRC. Colombia. https://forbes.co/2020/05/28/tecnologia/colombia-tiene-las-condiciones-paraimpulsar-el-5g-crc/

- MINTIC, (2019). plan $\quad 5 \mathrm{~g}$ Colombia. https://www.mintic.gov.co/portal/604/articles118058_plan_5g_2019120.pdf

- Xirio Online. (2020). Manual Xirio Online. http://www.xirioonline.com/help/es/index.htm?frequency_band.htm.

- Jyrki TJ P. (2019). O'Reilly library. Wiley. EEUU. http://www.oreilly.com /library/viw/5gexplained/9781118275688/ 
- Ceron, C. E, Vejarano S, M, C. (2018). Análisis de la capacidad de una red celular utilizando MMWAVE. http://repositorio.unicauca.edu.co:8080/bitstream/handle /123456789/1644/AN\%c3\%81LISIS\%20DE\%20LA\%20CAPACIDAD\%20DE\%20UNA \%20RED\%20CELULAR\%205G\%20HACIENDO\%20USO\%20DE\%20MMWAVE.pdf? sequence $=1 \&$ isAllowed $=\mathrm{y}$

- Martinez, A, R. (2017). Planificación de sistemas de radio comunicación en la banda de 3.5 $\mathrm{GHz}$ para la utilización de comunicaciones móviles 5G. http://oa.upm.es/52780/1/TFG_RICARDO_ALONSO_MARTINEZ.pdf

- Procedimiento de cálculo de la recomendación UIT-R P1411 en la herramienta XirioOnline, disponible en: https://www.xirio-online.com/help/es/rec_1411htm

David R. (2016). 5G asi es la tecnología que acelera tu móvil en el 2020. Tuexperto.com. 